\title{
Vitamin D binding globulin levels and affinity in various clinical conditions
}

\author{
IRF BROWN,* ANITA SOOD,* AND ND CARTER $\dagger$ \\ From the Departments of *Chemical Pathology and $\dagger$ Child Health, St George's Hospital Medical School, \\ Cranmer Terrace, London SWI7 ORE, UK
}

SUMMARY The approximate association constants of the plasma vitamin $\mathrm{D}$ binding globulin (Gc-globulin) for 25-hydroxycholecalciferol $\left(25(\mathrm{OH}) \mathrm{D}_{3}\right)$ and the plasma $25(\mathrm{OH}) \mathrm{D}_{3}$ binding capacities were measured in samples from 123 patients with a variety of disorders. No gross differences in binding affinities were observed between different groups of patients and controls. Many patients, however, had moderately reduced, and several had grossly reduced, plasma binding capacities. The changes in Gc-globulin relative to some other proteins are also described in detail in three patients during the course of their illness. Gc-globulin concentration and hence plasma vitamin $\mathrm{D}$ binding capacity can undergo rapid and marked changes during illness.

In common with most steroids, vitamin $\mathrm{D}$ (cholecalciferol) and its metabolites circulate in human plasma bound to protein. Recently, it has become apparent that this vitamin D binding protein is identical with group-specific component protein, Gc-globulin. ${ }^{1}$ Although this $\alpha_{2}$-globulin was first detected over 20 years ago, ${ }^{2}$ most studies on it have centred on its value as a genetic marker since two common codominant autosomal alleles, Gc1 and $\mathrm{Gc2}$, are found in all populations. With the assignment of a function to the products of these alleles there is an increased interest in the relationship between their properties and concentrations and disorders of calcium metabolism. There appears to be little difference in the vitamin $D$ binding properties of plasma from healthy individuals with the three normal phenotypes Gc1-1, Gc2-1, and Gc2-2. ${ }^{3}$ Gc-globulin is synthesised in the liver, ${ }^{4}$ and plasma concentrations generally fall to a moderate extent in chronic liver disease. ${ }^{5}$

However, no studies as yet seem to have been carried out on possible changes in the plasma vitamin D binding properties in disease. Furthermore, we occasionally have found very low concentrations of Gc-globulin in acutely ill patients. These observations, therefore, have prompted us to examine the vitamin $\mathrm{D}$ binding properties of plasma from patients with a variety of disorders and to record in a few selected patients the changes in concentrations of Gc-globulin that occur during the course of their illness.

Received for publication 12 February 1980

\section{Patients and methods}

Plasma samples for measurement of 25-hydroxycholecalciferol $\left(25(\mathrm{OH}) \mathrm{D}_{3}\right)$ binding affinity were selected from those received in the routine chemical pathology laboratory over a five-month period. Samples were selected mainly on the basis of abnormal results for plasma chemistry which, in conjunction with the clinical diagnosis on the request form, suggested that the affected patient had a condition that could possibly give rise to changes in the metabolism of vitamin D and its binding globulin. Thus the samples selected included those from patients with $(a)$ hypocalcaemia $(\mathrm{Ca}<1.85$ $\mathrm{mmol} / \mathrm{l}$ ) associated with renal failure (urea $>20$ $\mathrm{mmol} / \mathrm{l}, \mathrm{n}=22)$, vitamin $\mathrm{D}$ deficiency $(\mathrm{n}=3)$, thyroidectomy $(n=3)$, the newborn $(n=10)$, and a variety of other disorders including liver failure $(\mathrm{n}=23) ; \quad(b)$ hypercalcaemia $(\mathrm{Ca}>3.0 \mathrm{mmol} / \mathrm{l})$ associated with primary hyperparathyroidism $(n=5)$, bone secondaries $(n=6)$, and a variety of other disorders $(n=10)$; and $(c)$ calcium concentrations that were normal $(2 \cdot 15-2 \cdot 70 \mathrm{mmol} / \mathrm{l})$ and included patients who had thyrotoxicosis $(n=10)$, hypothyroidism $(n=6)$, liver disease $(n=6)$, hypophosphataemia (phosphate $<0.6 \mathrm{mmol} / \mathrm{l}, \mathrm{n}=8$ ), and other miscellaneous disorders $(n=11)$. Control samples $(n=18)$ were obtained from laboratory staff.

SCATCHARD PLOTS

The approximate association constants, $K_{a}$, of Gc-globulin for $25(\mathrm{OH}) \mathrm{D}_{3}$ were determined by a screening procedure involving a three-point 
Scatchard plot. For each plasma sample, three reaction tubes were set up containing respectively $0,0.5$, and $1.5 \mathrm{pmol}$ of unlabelled $25(\mathrm{OH}) \mathrm{D}_{3}$ dissolved in ethanol. After evaporation of the ethanol to dryness, 25-hydroxy(26(27)-methyl- $\left.{ }^{3} \mathrm{H}\right)$ cholecalciferol (Radiochemical Centre, Amersham, specific activity $9 \cdot 0-11 \cdot 8 \mathrm{Ci} / \mathrm{mmol}$ ) also dissolved in ethanol $(0.04 \mathrm{ml}$ containing 1.8-2.5 pmol) was pipetted out into the tubes. To each assay tube was added $0.4 \mathrm{ml}$ of plasma diluted $1: 800$ with phosphate buffer $(0.05 \mathrm{~mol} / 1$ containing gelatin, $200 \mathrm{mg} / \mathrm{l}$, pH 7.6) and the solutions were incubated for 2 hours at $4^{\circ} \mathrm{C}$. Non-specific binding was obtained by incubating buffer instead of diluted plasma. At the end of the incubation period $0.2 \mathrm{ml}$ of dextrancharcoal (1.25 g Norit GSX (Hopkin and Williams Ltd) and $0.125 \mathrm{~g}$ dextran (Koch-Light, MW 60000 90000 ) in $100 \mathrm{ml} \mathrm{H}_{2} \mathrm{O}$ ) was added, and after a further 20 minutes at $4{ }^{\circ} \mathrm{C}$ the tubes were centrifuged; $0.4 \mathrm{ml}$ of the supernatant was removed, and the radioactivity was counted in a modified Bray's scintillation solution. ${ }^{6}$ The results were analysed as Scatchard plots ${ }^{7}$ and the association constants $\left(\mathrm{K}_{\mathbf{a}}\right)$ and binding capacities (Cap) were calculated. ${ }^{8}$

\section{PROTEIN MEASUREMENTS}

Specific proteins were measured in some of the samples used in the screening procedure and also in a few selected patients during the course of their illness. Transferrin, caeruloplasmin, and Gc-globulin were measured using M-Partigen immunodiffusion plates (Hoechst Pharmaceuticals) containing the appropriate antiserum. Albumin was measured by a bromocresol green dye-binding method (Technicon Method AAII No. 30).

\section{Results}

The Table shows the association constants, $K_{a}$, for the binding of $25(\mathrm{OH}) \mathrm{D}_{3}$ to Gc-globulin and the plasma $25(\mathrm{OH}) \mathrm{D}_{3}$ binding capacities, Cap, obtained in plasma samples from patients with a variety of disorders. In no group of patients did the mean $\mathrm{K}_{\mathrm{a}}$ values differ significantly from that obtained in the controls. However, the plasma binding capacities in most groups of patients were significantly lower than in the controls.

The $25(\mathrm{OH}) \mathrm{D}_{3}$ binding capacity should be a measure of the concentration of Gc-globulin in plasma. That this was so was tested by measuring Gc-globulin concentrations by radial immunodiffusion in plasma samples selected so as to give a wide range of binding capacities. Figure 1 shows the relationship between these two independent estimates of vitamin D binding globulin. A highly significant correlation was found $(r=0.78$, $P<0.001)(y=14.7 x-0.34)$.

During the course of these screening procedures it became obvious that there was considerable variation in the vitamin $D$ binding capacity of samples from different patients. Figure 2 shows that, although there was a significant correlation between binding capacity and albumin $(r=0.53, \mathrm{P}<0.001)$, there was in the samples assayed roughly a 10 -fold variation in the former compared with only a twofold variation in the latter. This implies that the concentration of Gc-globulin in human plasma is rather more variable than that of albumin.

These observations, therefore, prompted us to examine the temporal relationships between Gcglobulin and some other proteins during the course

25-hydroxy vitamin $D$ association constants $\left(K_{\mathrm{a}}\right)$ and plasma binding capacities $(C a p)$ in various groups of patients

\begin{tabular}{|c|c|c|c|}
\hline & No. & $K_{\mathrm{a}}\left(\times 10^{-} \cdot \mathrm{l} / \mathrm{mol} \pm S E M\right)$ & $\operatorname{Cap}(\mu m o l / l \pm S E M)$ \\
\hline \multicolumn{4}{|l|}{ Hypocalcaemia $(\mathrm{Ca}<1.85 \mathrm{mmol} / \mathrm{l})$} \\
\hline Renal failure & 22 & $0.74 \pm 0.07$ & $* 3.26 \pm 0.32$ \\
\hline Vitamin D deficiency & 3 & $0.90 \pm 0.25$ & $* 2.28 \pm 0.38$ \\
\hline Premature infants & 10 & $1 \cdot 10 \pm 0 \cdot 19$ & $* 2.53 \pm 0.31$ \\
\hline Others & 26 & $0.74 \pm 0.06$ & $* 3.16 \pm 0.23$ \\
\hline \multicolumn{4}{|l|}{ Hypercalcaemia $(\mathrm{Ca}>3.0 \mathrm{mmol} / \mathrm{l})$} \\
\hline Primary hyperparathyroidism & 5 & $0.77 \pm 0.13$ & $4.05 \pm 0.48$ \\
\hline Bone secondaries & 6 & $0.77 \pm 0.12$ & $* 3.09 \pm 0.45$ \\
\hline Others & 10 & $0.94 \pm 0.15$ & $* 3.47 \pm 0.27$ \\
\hline \multicolumn{4}{|c|}{ Normocalcaemia $(\mathrm{Ca}=2 \cdot 15-2 \cdot 70 \mathrm{mmol} / \mathrm{l})$} \\
\hline Thyrotoxicosis & 10 & $0.89 \pm 0.12$ & $\dagger 3.61 \pm 0.29$ \\
\hline Hypothyroid & 6 & $1 \cdot 12 \pm 0 \cdot 14$ & $4 \cdot 26 \pm 0.39$ \\
\hline Liver disease & 6 & $0.69 \pm 0.08$ & $+3.36 \pm 0.48$ \\
\hline Hypophosphataemia & 8 & $0.84 \pm 0.14$ & $* 3.44 \pm 0.33$ \\
\hline Others & 11 & $0.61 \pm 0.13$ & $4 \cdot 15 \pm 0.37$ \\
\hline Controls & 18 & $0.87 \pm 0.08$ & $4.91 \pm 0.38$ \\
\hline
\end{tabular}

Significant difference between patients and controls, ${ }^{*} \mathbf{P}<0.01, \dagger_{P}<0.02$. 


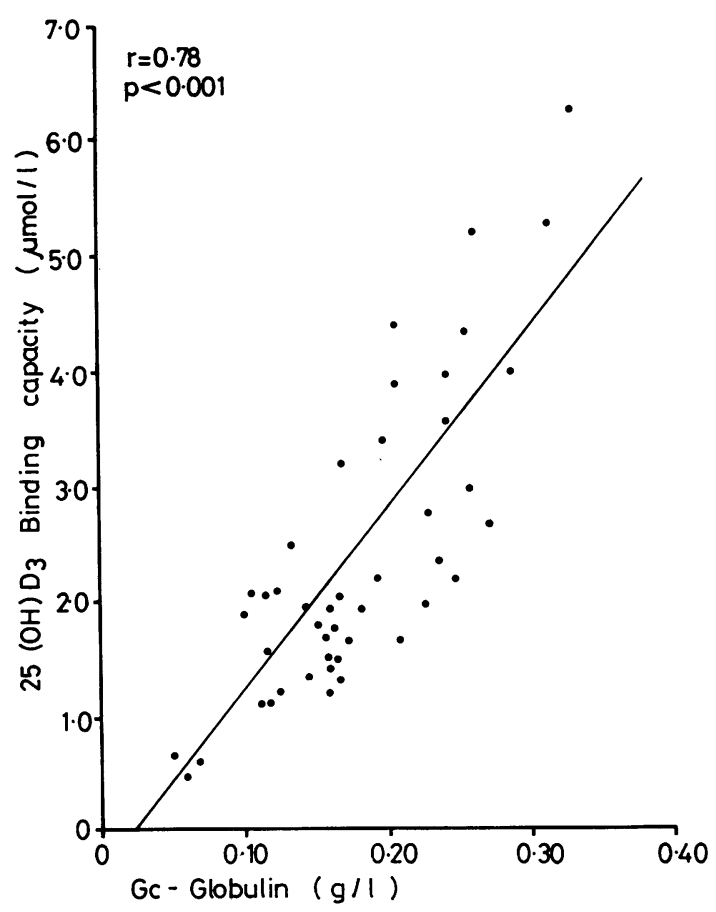

Fig. 1 Relationship between plasma $25(\mathrm{OH}) \mathrm{D}_{3}$ binding capacity $(\mu \mathrm{mol} / \mathrm{l})$ as determined by the Scatchard screening procedure and Gc-globulin determined by radial immunodiffusion.

of individual patients' illnesses. Three patients were selected. The changes in Gc-globulin and in caeruloplasmin, transferrin, and albumin are shown in Figure 3. Results are expressed as a percentage of the preoperative protein concentration or the protein concentration on admission. All four proteins were within normal limits in the patients when samples were obtained initially.

CASE 1

A 41-year-old man had three parathyroid adenomata removed. His postoperative course was uneventful until the last blood sample taken on day 15. In this patient transferrin and albumin concentrations fell postoperatively and had not quite regained the preoperative concentrations by day 15 . Caeruloplasmin concentration rose from day 3 to day 8 and was still elevated after 15 days. Gc-globulin paralleled the changes in caeruloplasmin from day 1 to day 6 but subsequently its concentration fell and by day 15 was only $10 \%$ above the day 0 value. Thus, in this patient, the Gc-globulin is acting like an 'acute phase reactant' but with a response that is less

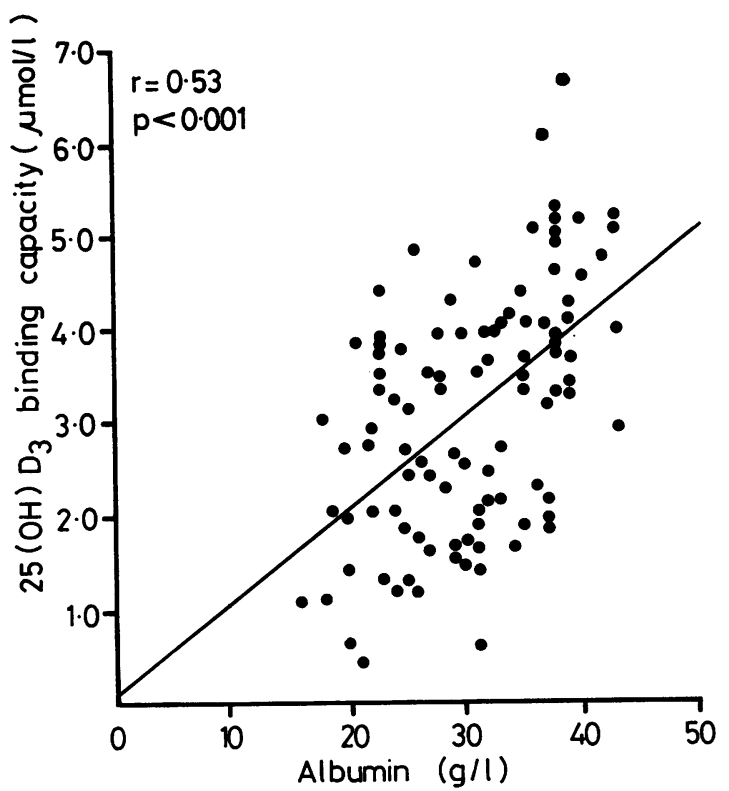

Fig. 2 Relationship between plasma $25(\mathrm{OH}) \mathrm{D}_{3}$ binding capacity $(\mu \mathrm{mol} / \mathrm{l})$ and albumin $(\mathrm{g} / \mathrm{l})$.

sustained than the more typical response of caeruloplasmin.

\section{CASE 2}

A 67-year-old man was admitted with a suspected and subsequently confirmed myocardial infarction. There was no extension of the infarct; the patient improved rapidly and was eventually discharged 13 days after admission. Again, in this patient, changes in albumin and transferrin paralleled one another, and concentrations were depressed by 20 to $40 \%$ of those obtained on admission. Caeruloplasmin responded once more like a typical 'acute phase reactant', and the concentration continued to rise throughout the 11 days of observation. By day 11 it was $70 \%$ above the admission value. In contrast, Gc-globulin concentrations showed no consistent trend and varied within $\pm 11 \%$ of the admission value.

\section{CASE 3}

A 17-year-old girl was admitted in a sickle cell crisis with a concomitant pulmonary infection. She was treated in hospital for $\mathbf{2 3}$ days. This patient was acutely ill for the first six days after admission, during which period she received 4 units each of whole blood and fresh frozen plasma and was losing about $20 \mathrm{~g}$ of protein per day in her urine. This obviously complicates the interpretation of serum 


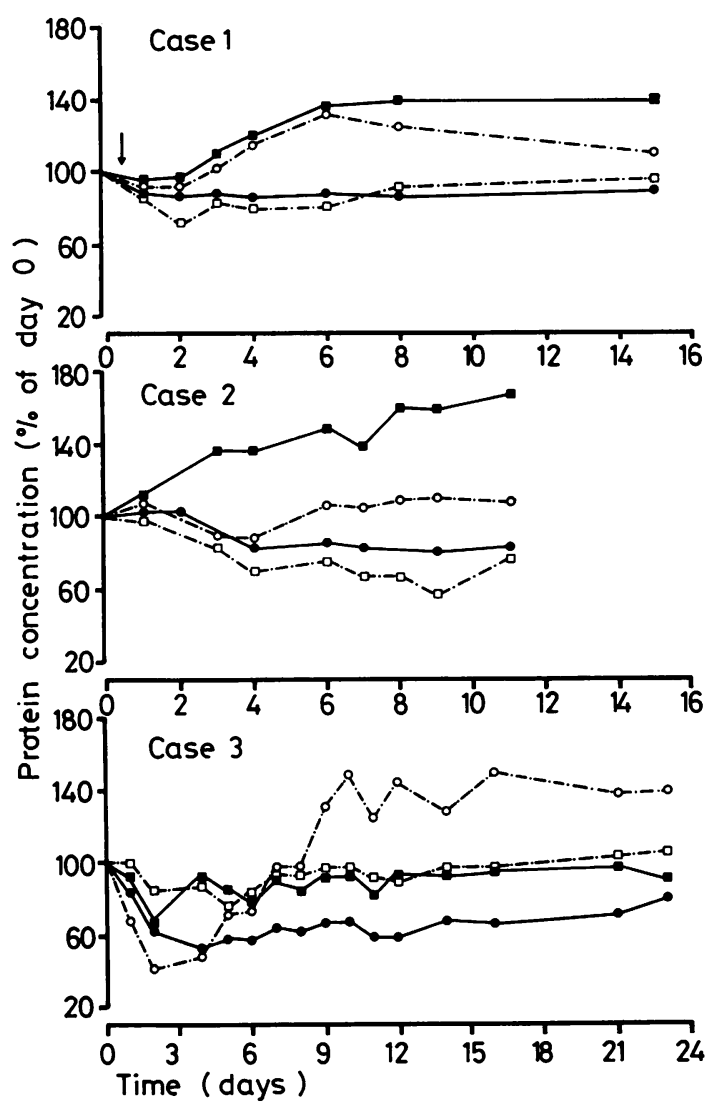

Fig. 3 Changes in serum concentrations of albumin (-O), Gc-globulin ( $\bigcirc---\bigcirc)$, caeruloplasmin ( $\square-\square)$, and transferrin $(\square---\square)$, in three patients during the course of their illnesses. Case 1, parathyroidectomy; case 2, myocardial infarction; case 3, sickle cell crisis.

protein measurements but nevertheless it is quite clear that the Gc-globulin changed in a markedly different fashion from the other three proteins. The concentrations of all four proteins were depressed in the first few days, but the Gc-globulin fell to the lowest level at $40 \%$ of that seen on admission. From day 4 onwards there was a slow rise in transferrin, caeruloplasmin, and albumin, the transferrin and caeruloplasmin concentrations by day 9 being close to those found on admission, though the albumin remained depressed for the next three weeks. However, in contrast, there was a remarkable 'overshoot' in the Gc-globulin concentration, and between day 2 and day 10 it changed from $40 \%$ to $150 \%$ of the admission value. It then remained at this high level for the next two weeks. The patient was not taking oestrogen preparations, which would have affected the protein concentrations. Her Gc-globulin level on discharge was still on a plateau at about $30 \%$ above the top end of our reference range.

\section{Discussion}

The object of the present study was to try to get further information on the role, if any, that Gcglobulin might play in the pathogenesis of disorders of mineral metabolism. Previous studies have shown that Gc-globulin concentrations are depressed in chronic liver disease ${ }^{5}$ but that the concentrations remain within the normal range in a variety of situations, including vitamin D deficiency, vitamin D therapy, sarcoidosis, osteoporosis, and thyrotoxicosis. ${ }^{910}$ In common with many other proteins, the concentrations are increased by pregnancy and oestrogen preparations. Increases have also been reported in haemochromatosis ${ }^{11}$ and chronic active hepatitis. ${ }^{12}$

The only function so far ascribed to Gc-globulin is that of transporting vitamin D metabolites. However, at normal physiological concentrations of $25(\mathrm{OH}) \mathrm{D}_{3}(25-100 \mathrm{nmol} / \mathrm{l})$, the binding capacity of human plasma is only about $3 \%$ saturated. Thus it would appear that only a major decrease in circulating Gc-globulin would be sufficient to interfere with vitamin D transport. Previous reports indicate that the reductions are not severe enough to account for the disturbances in bone metabolism that occur in chronic liver disease. 5

In our present survey three out of the 123 patients studied had plasma Gc-globulin concentrations less than $0 \cdot 10 \mathrm{~g} / 1$ (reference range $0 \cdot 25-0.35 \mathrm{~g} / \mathrm{l}$ ). By chance, results for transferrin and caeruloplasmin concentrations were also available on these patients and were found to be normal in two and moderately reduced in the third. In four other patients not included in the survey, we have observed comparable low Gc-globulin concentrations. All seven of these patients were severely ill, but the lowest value recorded $(0.059 \mathrm{~g} / \mathrm{l})$ still accounts for about $20 \%$ of the vitamin D binding capacity of normal serum.

If alterations in the circulating concentrations of Gc-globulin seem unlikely to play a role in abnormalities of calcium metabolism, it is possible that changes in the binding affinity of vitamin $\mathbf{D}$ metabolites for the protein in pathological sera might do so. The screening procedure described in this study was designed so as to enable us to check whether gross changes in binding affinity did in fact occur. In samples selected from various groups of patients we were unable to detect any significant deviation of the binding affinity from normal. It, therefore, seems unlikely that there are gross differences in the 
intravascular transport of $25(\mathrm{OH}) \mathrm{D}_{3}$ between patients who have or are at risk of developing abnormalities of calcium metabolism. Our present technique, however, does not exclude the possibility that minor differences might exist between patients with different illnesses as has been described, for example, for cortisol binding globulin affinity in anorecticts. ${ }^{13}$

What is clear is that during illness individual patients may show quite marked variations in Gc-globulin concentrations. Gc-globulin has previously been reported to behave rather like caeruloplasmin in that slow and moderate increases are seen in patients after surgery. ${ }^{14}$ However, other workers have suggested that it is not an acute phase reactant, levels remaining normal in conditions with acute inflammation or chronic infections. ${ }^{15}$

The results from the three patients whom we have studied suggest that the physiological response to trauma has a somewhat variable effect on plasma Gc-globulin concentration. It can lead to an increase in concentration in a moderate acute phase reaction rather similar to that seen for caeruloplasmin (case 1). There may be little change (case 2). Finally, there may be very rapid alterations in Gc-globulin concentration (case 3). The explanation of these changes in Gc-globulin concentration is not clear. Concentration of a protein in plasma is dependent on a variety of factors, including rate of synthesis, rate of degradation, and distribution between intravascular and extravascular pools. The findings that some acutely ill patients can have very low Gcglobulin relative to other proteins and that the rate of change of concentration can also be rapid seems to suggest that Gc-globulin has a relatively high turnover rate. Furthermore, the pattern of response of Gc-globulin to trauma can be independent of the response given by other plasma proteins.

We thank the Medical Research Committee, St George's Hospital and the South West Thames Regional Health Authority for financial support. $25(\mathrm{OH}) \mathrm{D}_{3}$ was kindly supplied by Dr JW Hinman, of the Upjohn Company, Kalamazoo, Michigan, USA.
References

${ }^{1}$ Daiger SP, Schanfield MS, Cavalli-Sforza LL. Groupspecific component $(\mathrm{Gc})$ proteins bind vitamin $\mathrm{D}$ and 25-hydroxy-vitamin D. Proc Nat Acad Sci USA 1975; 72:2076-80.

${ }^{2}$ Hirschfeld J. Immune-electrophoretic demonstration of qualitative differences in human sera and their relation to haptoglobins. Acta Path Microbiol Scand 1959;47: 160-8.

${ }^{3}$ Brown IRF, Carter ND, Sood A. Vitamin D binding globulin phenotypes in liver disease. Clin Chim Acta 1979;95:75-82.

4 Prunier JH, Bearn AG, Cleve $\mathrm{H}$. Site of formation of the group-specific component and certain other serum proteins. Proc Soc Exp Biol Med 1964;115:1005-7.

${ }^{5}$ Barragry JM, Corless D, Auton J, Carter ND, Long RG, Maxwell JD, Switala S. Plasma vitamin D-binding globulin in vitamin $\mathrm{D}$ deficiency, pregnancy and chronic liver disease. Clin Chim Acta 1978;87:359-65.

- Bray GA. A simple efficient liquid scintillator for counting aqueous solutions in a liquid scintillation counter. Anal Biochem 1960;1:279-85.

${ }^{7}$ Scatchard G. The attractions of proteins for small molecules and ions. Ann NY Acad Sci 1949;51:660-72.

${ }^{8}$ Rosenthal HE. A graphic method for the determination and presentation of binding parameters in a complex system. Anal Bioch 1967;20:525-32.

${ }^{9}$ Haddad JG, Walgate J. Radioimmunoassay of the binding protein for vitamin $D$ and its metabolites in human serum. J Clin Invest 1976;58:1217-22.

${ }^{10}$ Bouillon R, Van Baelen H, De Moor P. The measurement of the vitamin D-binding protein in human serum. $J$ Clin Endocrinol Metab 1977;45:225-31.

1 Amin AH, Clarke HG, Freeman T, Murray-Lyon M, Smith PM, Williams R. Studies by quantitative immunoelectrophoresis on iron binding proteins in haemochromatosis. Clin Sci 1970;38:613-6.

${ }^{12}$ Mihas AA, Hirschowitz BI. Group-specific component (Gc) levels in chronic liver disease. J Med 1978;9:109-15.

${ }^{13}$ Casper RG, Chatterton RT, Davis JM. Alterations in serum cortisol and its binding characteristics in anorexia nervosa. J Clin Endocrinol Metab 1979;49:406-11.

14 Clarke HGM, Freeman T, Pryse-Phillips W. Serum protein changes after injury. Clin Sci 1971;40:337-44.

${ }^{15}$ Cleve $H$. The variants of the group-specific component. Isr J Med Sci 1973;9:1133-46.

Requests for reprints to: Dr IRF Brown, Department of Chemical Pathology, St George's Hospital Medical School, Cranmer Terrace, London SW17 0RE, UK. 